\title{
Congenital Aorto-pulmonary Fistula Combined with Persistent Ductus Arteriosus
}

\author{
E. N. COLEMAN, R. S. BARCLAY, J. M. REID, AND J. G. STEVENSON \\ From the Department of Cardiology and the University Department of Child Health, \\ Royal Hospital for Sick Children, Glasgow, and the Cardio-Thoracic Unit, Mearnskirk Hospital, Renfrewshire
}

Congenital aorto-pulmonary fistula is a relatively uncommon malformation (Dadds and Hoyle, 1949; Cooley, McNamara, and Latson, 1957; Neill and Mounsey, 1958; Parker et al., 1963) and for it to be accompanied by another aorto-pulmonary shunt through a persistent ductus arteriosus must be distinctively rare (Straus, Lev, and Cassels, 1964). In this description of two children with the unsuspected combination of these defects, the diagnostic and therapeutic problems are presented.

\section{CASE Reports}

Case 1. This baby girl, the younger of two sibs, was born after an uneventful pregnancy in August 1961, weighing $2.3 \mathrm{~kg}$. Her development and growth were satisfactory, but during an upper respiratory tract infection, at the age of 8 months, a heart murmur was detected. Respiratory infections were frequent and were characterized by many choking attacks. Breathlessness on feeding was reported between the ages of 2 and 12 months; there was no cyanosis at any time.

Clinical examination revealed a thin but active infant with collapsing pulses; the blood pressure (right arm) was $115 / 50 \mathrm{~mm}$. Hg. The apical impulse was well localized to the fifth left intercostal space $1 \mathrm{~cm}$. outside the nipple line; right ventricular pulsation was palpable. A continuous murmur characteristic of persistent ductus arteriosus was maximal and accompanied by a thrill at the pulmonary area; pulmonary valve closure was accentuated. The electrocardiogram indicated severe left and less severe right ventricular hypertrophy. Cardiomegaly on $x$-ray film (CTR $60 \%$ ), consisting of biventricular and left atrial enlargement, was accompanied by pulmonary vascular engorgement (Fig. 1a). Cardiac catheterization (Table I) disclosed a substantial increase in oxygen saturation in the pulmonary arteries, but the aorta was not entered. There was no pulmonary hypertension. A minor systolic pressure gradient across the pulmonary

Received June 30, 1966 valve was detected. Angiocardiography was considered unnecessary.

At thoracotomy in November 1962 a ductus arteriosus of no more than average width was ligated; the main pulmonary artery did not become substantially smaller and a systolic thrill persisted over the right ventricular outflow tract. The pericardium was not opened but a small ventricular septal defect was suspected. At the first outpatient examination 3 months later, no change was noted from the pre-operative physical signs. A transient decrease in the cardiac dimensions on $x$-ray film was followed during the next 18 months by a further increase (Fig. 1b). Electrocardiographic evidence of left ventricular hypertrophy persisted but now without evidence of right ventricular hypertrophy. The ductus was thought to have re-canalized, and thoracotomy was repeated 21 months later without further cardiac catheterization. The ductus was not patent but a fistula was discovered between ascending aorta and main pulmonary artery $0.5 \mathrm{~cm}$. in diameter and $1 \mathrm{~cm}$. above the valve. After it had been freed by dissection, double ligation with floss silk completely occluded its lumen. Blood oxygen saturation values now showed no evidence of any shunt, and the thrill over the right ventricular outflow tract had been abolished. The heart size (Fig. 1c) gradually reduced (CTR from $63 \%$ to $59 \%$ ) in the following 8 months; the blood pressure, which before the second operation had been $110 / 50 \mathrm{~mm}$. $\mathrm{Hg}$, now measured $95 / 75 \mathrm{~mm} . \mathrm{Hg}$, and only a short soft systolic murmur persisted at the pulmonary area.

TABLE I

CARDIAC CATHETERIZATION DATA (CASE 1)

\begin{tabular}{l|c|c}
\hline \multicolumn{1}{c|}{ Site } & $\begin{array}{c}\text { Oxygen saturation } \\
(\%)\end{array}$ & $\begin{array}{c}\text { Pressure } \\
(\mathrm{mm} . \mathrm{Hg})\end{array}$ \\
\hline $\begin{array}{l}\text { Right atrium } \\
\text { Right ventricle }\end{array}$ & 58 & - \\
$\begin{array}{l}\text { Main pulmonary } \\
\text { artery }\end{array}$ & 63 & $38 / 0$ \\
$\begin{array}{l}\text { Right main } \\
\text { pulmonary artery }\end{array}$ & 78 & $20 / 12$ \\
\hline
\end{tabular}




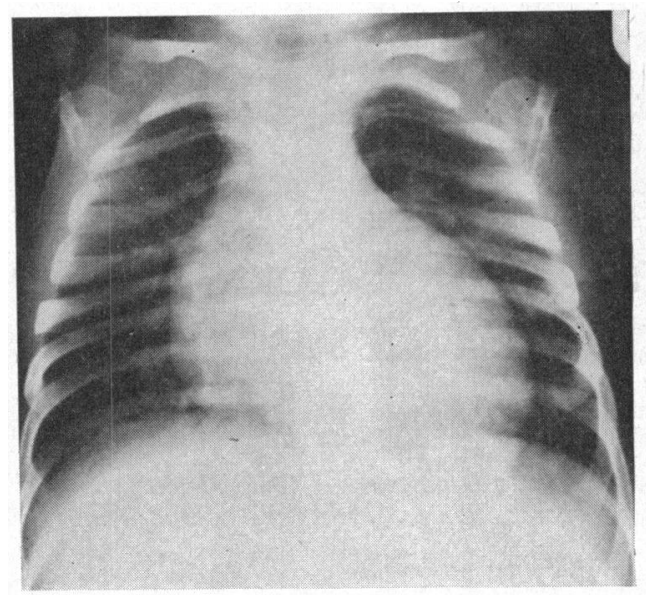

(a)

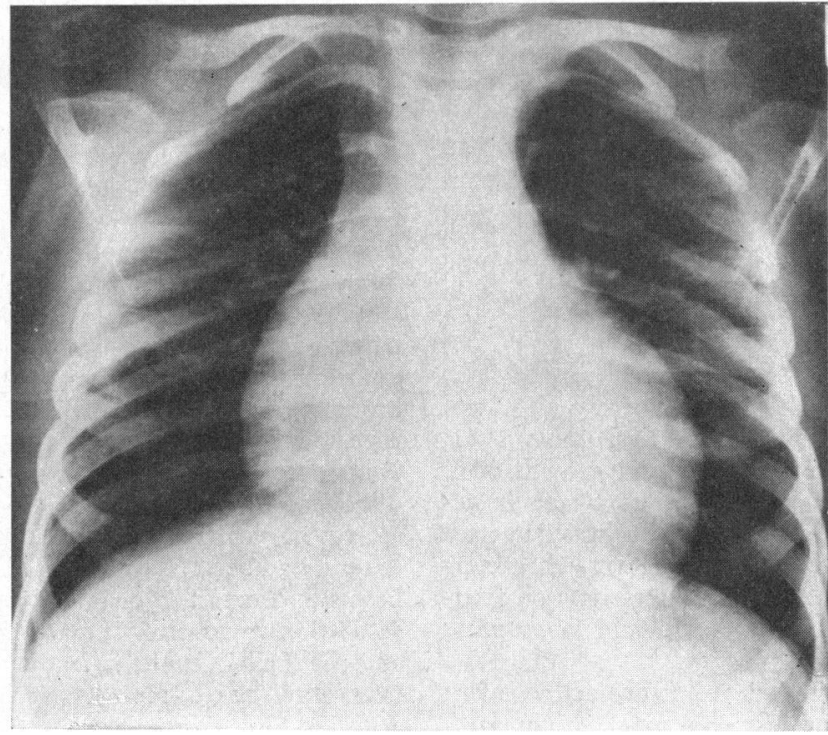

(c)

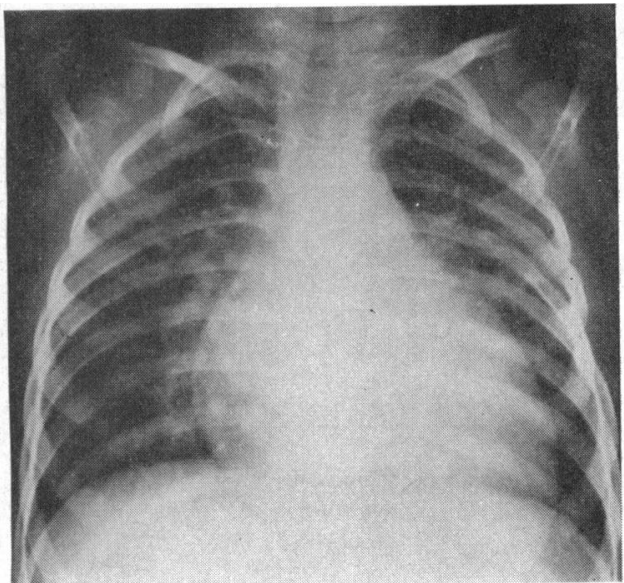

(b)

(b) 11 months after (CTR 64\%);

FIG. 1.-Case 1. (a) Immediately before ligating ductus (CTR 60\%); (b) 11 months
(c) 6 months after closure of aorto-pulmonary fistula (CTR 59\%).

Case 2. This girl, the last of eight sibs, was born after an uneventful pregnancy in December 1961, weighing $2.5 \mathrm{~kg}$. During bronchopneumonia at the age of 8 weeks, a heart murmur was discovered. Thereafter, though slow to feed, she was not unduly breathless on exertion and there was no central cyanosis. From the age of 5 months frequent attacks of bronchitis began, leading to increasing thoracic deformity and decreasing exercise tolerance.

Clinical examination at 5 months revealed a vigorous infant, apparently thriving, with collapsing pulses; the blood pressure (right arm) was recorded as $130 / 50$ $\mathrm{mm}$. Hg. The heart was much enlarged, a diffuse apical impulse being palpable in the sixth left intercostal space in the mid-axillary line; right ventricular pulsation was pronounced. The second sound at the pulmonary area was very loud, and a loud harsh pansystolic murmur along the left sternal border was maximal towards its lower extremity. The electrocardiogram indicated severe bi-ventricular hypertrophy with a dominant left ventricle. Cardiomegaly (CTR 63\%) was revealed by $x$-ray film (Fig. 2a), with enlargement of both ventricles and pulmonary vascular engorgement. Cardiac catheterization (Table II) demonstrated severe pulmonary hypertension accompanying a substantial arteriovenous shunt at pulmonary artery level; frequently the catheter 


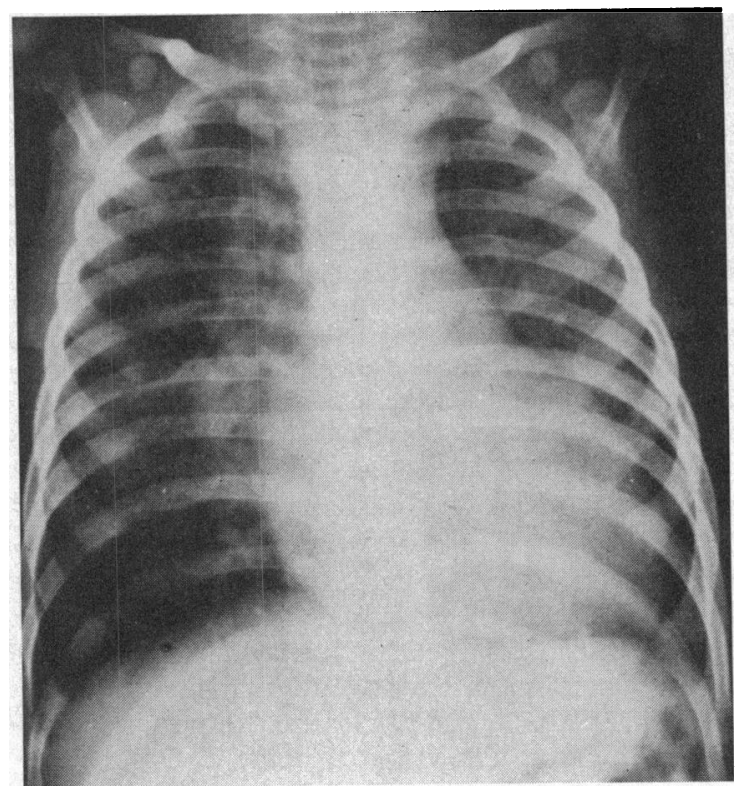

(a)

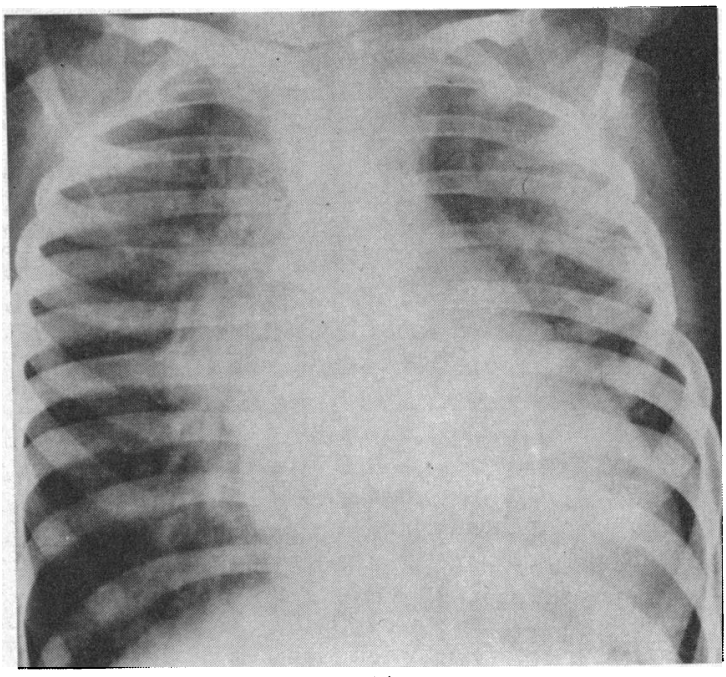

(c)

FIG. 2.-Case 2. (a) Immediately before ligation of ductus (CTR 63\%); (b) 3 years after (CTR 66\%); (c) 6 months after closure of aorto-pulmonary fistula (CTR 63\%).

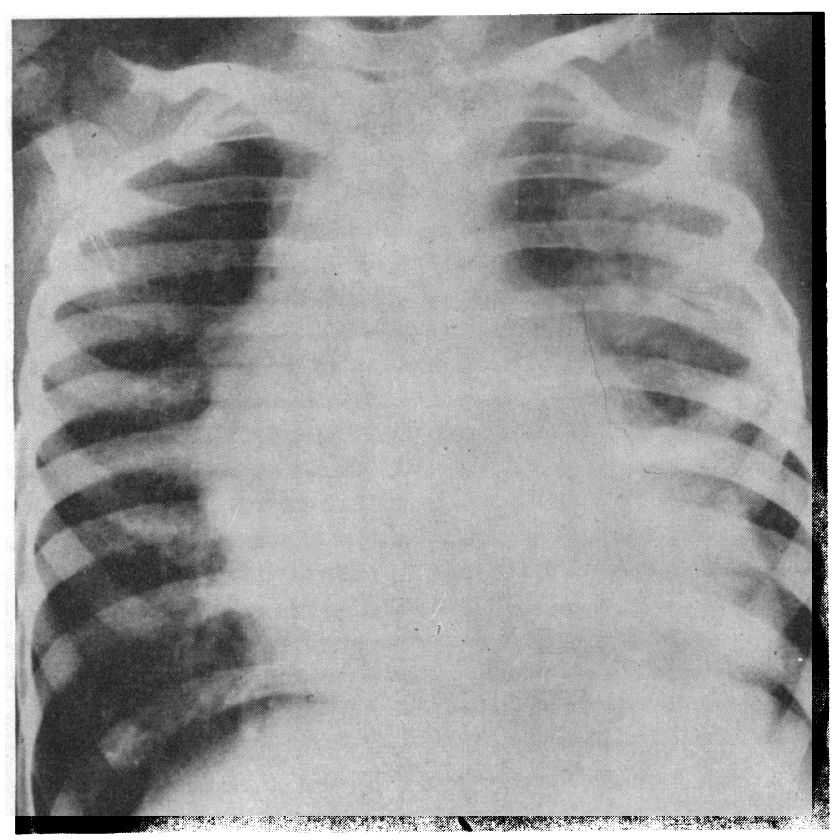

(b)

traversed a communicating vessel between main pulmonary artery and aorta, which had every appearance of being the ductus arteriosus (Fig. 3).

At thoracotomy in June 1962, a ductus arteriosus of average size was ligated, but this did not to any extent influence the pulmonary artery pressure, and a thrill over the base of the heart persisted. During the following 
TABLE II

CARDIAC CATHETERIZATION DATA (CASE 2)

\begin{tabular}{|c|c|c|c|c|}
\hline \multirow{2}{*}{ Site } & \multicolumn{2}{|c|}{$\stackrel{\text { First }}{\text { catheterization }}$} & \multicolumn{2}{|c|}{$\begin{array}{l}\text { Second } \\
\text { catheterization }\end{array}$} \\
\hline & $\begin{array}{c}\text { Oxygen } \\
\text { saturation } \\
(\%)\end{array}$ & $\begin{array}{c}\text { Pressure } \\
(\mathrm{mm} . \mathrm{Hg})\end{array}$ & $\begin{array}{c}\text { Oxygen } \\
\text { saturation } \\
(\%)\end{array}$ & $\begin{array}{l}\text { Pressure } \\
(\mathrm{mm} . \mathbf{H g})\end{array}$ \\
\hline $\begin{array}{l}\text { Right atrium } \\
\text { Right ventricle }\end{array}$ & $\begin{array}{l}54 \\
54\end{array}$ & $\overline{84 / 0}$ & $\begin{array}{l}59 \\
59\end{array}$ & $\overline{72 / 0}$ \\
\hline $\begin{array}{l}\text { Main pulmonary } \\
\text { artery }\end{array}$ & 86 & $80 / 30$ & 74 & $70 / 50$ \\
\hline $\begin{array}{l}\text { Right main } \\
\text { pulmonary artery }\end{array}$ & 85 & - & 72 & - \\
\hline
\end{tabular}

artery, but this seemed smaller than before. Pulmonary hypertension persisted but had not increased. The catheter could not, on this occasion, be advanced into the aorta from the pulmonary artery. Angiocardiography was not done because severe laryngospasm followed by a generalized convulsion developed approximately 30 minutes after the intravenous test dose of contrast medium. At the second thoracotomy, the ductus was not patent, but when the pericardium was opened, a large aorto-pulmonary fistula was revealed and, after lengthy dissection, isolated. The intention was to divide the fistula and repair both pulmonary artery and aorta. However, troublesome bleeding was encountered from the posterior aspect of the fistula towards the end of the dissection, and to control this and obliterate the fistula,

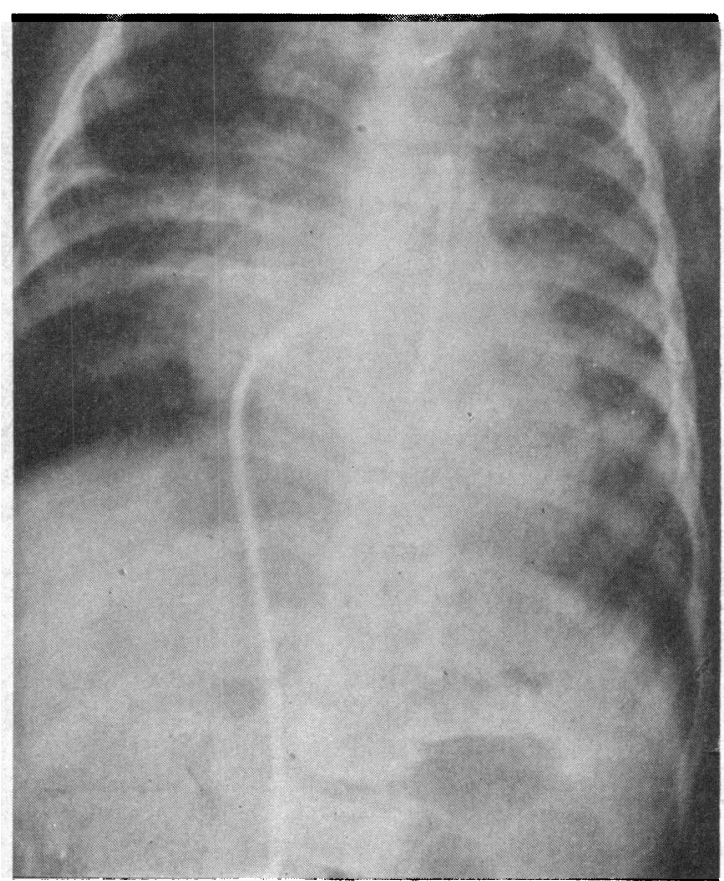

Fig. 3.-Case 2. Catheter advanced from main pulmonary artery to abdominal aorta through ductus arteriosus. several stitches were passed around this area. This produced abolition of the thrill but without affecting the level of pulmonary arterial hypertension. Her physical condition immediately after operation was precarious but responded to digitalization and diuretic therapy. During the next six months the heart size decreased (CTR 73\% to $63 \%$ ) but evidence of pulmonary hypertension persisted (Fig. 2c). There was still a systolic murmur down the left sternal border, and the systemic blood pressure remained unchanged both before and after the second operation at $95 / 60 \mathrm{~mm}$. Hg. Although electrocardiographic evidence of ventricular hypertrophy disappeared, one must conclude that the fistula was not completely obliterated at the second operation and that there is still a shunt at this level.

\section{Discussion}

Aorto-pulmonary fistula or septal defect results from a failure of the base of the spiral septum to develop and to complete the division of the fotal truncus arteriosus into aorta and pulmonary artery (Taussig, 1960). Situated close to the aortic root it may extend from the aortic cusps for a distance of from 5 to $30 \mathrm{~mm}$.; its area is believed to determine not only the size of the shunt but the degree of pulmonary hypertension and the prognosis (Nadas, 1963). Wood (1960) stated that it was clinically and physiologically indistinguishable from a persistent ductus arteriosus and likely to be encountered in 1-2 per cent of patients submitted for ductus ligation. In 1958, however, Skall-Jensen had been able to find only 62 cases previously reported. The combination of an aorto-pulmonary fistula with a persistent ductus arteriosus appears to be even less common. Straus et al. (1964) reported one such case in a young man with severe pulmonary hypertension who died during an operation to close an aorto-pulmonary fistula and whose persistent ductus arteriosus was discovered only at necropsy. In 340 consecutive operations for the closure of a persistent ductus, we have not once encountered an aortopulmonary fistula as the sole malformation present, but we have twice encountered it combined with a persistent ductus arteriosus and these two instances are the subject of our present communication.

Certain clinical features may help to distinguish an aorto-pulmonary fistula from a persistent ductus (Cooley et al., 1957; Parker et al., 1963). In contrast to the symptom-free patient with an uncomplicated persistent ductus, the patient with a fistula is expected to have symptoms including exertional dyspnoea, recurrent respiratory infections, and hæmoptysis. Other features include an unusually low site for the continuous murmur, $x$-ray evidence of severe cardiomegaly with pulmonary artery enlargement, and electrocardiographic evidence of biventricular hypertrophy. If angiocardiographic 
contrast medium is injected into the right ventricle, the dilution effect from aortic blood entering the pulmonary artery is expected to be visible at a lower level and further to the right than with a ductus arteriosus.

Both of our patients did, indeed, have frequent attacks of bronchitis, with coughing and spasms of choking being especially remarked upon in one case, but exertional dyspnoea of cardiac origin was noted only briefly during the first year of life and hæmoptysis not at all. The murmur of one patient (Case 1) was typical of a ductus arteriosus both before and after ligation of the ductus, whereas in the other (Case 2) it was confined to systole and was loudest at the lower sternal border. Both the timing and site of this murmur were regarded as being quite consistent with a ductus arteriosus complicated by severe pulmonary hypertension (Reid et al., 1964), but its persistence after operation was incorrectly attributed to continued patency of the ductus. Although not very severe, the degree of cardiomegaly and of pulmonary artery enlargement on $x$-ray film in Case 1 was, in retrospect, out of keeping with a persistent ductus of only average size at operation. The even greater degree of enlargement disclosed in $x$-ray examination of Case 2 was attributed to severe pulmonary hypertension, but again in retrospect the suspicious feature here was that severe pulmonary hypertension should have accompanied a ductus arteriosus discovered at operation to be of only average size. Although electrocardiographic evidence of biventricular hypertrophy was not unexpected in Case 2, thought to have pulmonary hypertension associated with a persistent ductus, it was as unexpected in the other (Case 1) as was the disappearance of the evidence of right ventricular hypertrophy after the ductus had been closed.

Cardiac catheterization gave no evidence of the presence of two aorto-pulmonary shunts, and, in the patient whose descending aorta the catheter entered, the crossing was achieved through the ductus arteriosus. In neither patient was angiocardiography performed at the pre-operative catheterizations because a persistent ductus was incorrectly supposed to be the sole malformation present, and at the other catheterization of the second patient because of an alarming reaction to the test dose of contrast medium. The more important diagnosis to be made in these children was that of aortopulmonary fistula, and the critical time for this diagnosis was before the first thoracotomy in order that preparations for the use of cardio-pulmonary bypass might have been made in readiness for that operation. In these circumstances, angiocardiography might have provided valuable aid in demonstrating an unusually low site for the dilution effect on the stream of contrast medium in the pulmonary artery caused by the entry of aortic blood through the aorto-pulmonary fistula.

Suspicion of the possibility of an aorto-pulmonary fistula may thus be raised by certain features suggesting an atypical ductus arteriosus, and may lead to definitive diagnosis by angiocardiography, but a pre-operative diagnosis of a combination of these malformations presents a problem with no obvious solution. If this combination of lesions were not suspected before operation, the discovery at thoracotomy of a ductus arteriosus of only average or narrow calibre in association with substantial cardiomegaly, pulmonary hypertension, electrocardiographic evidence of biventricular hypertrophy, and especially if there is a history of recurrent severe respiratory infection and limitation of exercise tolerance, makes almost certain the presence of an additional lesion. If closure of the ductus arteriosus then has little or no effect on pulmonary artery size and tension, and a thrill persists over the base of the heart, the pericardium should be opened so that the aorta and pulmonary artery may be inspected from their origins. Only in exceptional cases does it seem likely, however, that an aorto-pulmonary fistula will be sufficiently narrow to allow complete closure by simple ligation, as in one of our patients. More often, to achieve complete closure will require a second operation for division of the structure and repair of the great artery walls with the support of cardio-pulmonary bypass.

\section{SUMMARY}

Two children with the unusual combination of aorto-pulmonary fistula and persistent ductus arteriosus, unsuspected before thoracotomy, are described. Although certain atypical features may be present, a clinical diagnosis of an isolated persistent ductus arteriosus, with or without pulmonary hypertension, is likely. Mention is made of those features which, at thoracotomy for the closure of a persistent ductus arteriosus, may suggest the possibility that this additional anomaly exists.

We thank Professor J. H. Hutchinson for access to patients admitted to his clinical care. We are indebted to Drs. S. P. Rawson and Elizabeth M. Sweet for $x$-ray examinations, to $\mathrm{Mr}$. Hugh Gray for illustrations, and to medical and surgical colleagues in the Cardiac Departments of both hospitals. 


\section{REFERENCES}

Cooley, D. A., McNamara, D. G., and Latson, J. R. (1957). Aorticopulmonary septal defect: diagnosis and surgical treatment. Surgery, 42, 101.

Dadds, J. H., and Hoyle, C. (1949). Congenital aortic septal defect. Brit. Heart f., 11, 390.

Nadas, A. S. (1963). Pediatric Cardiology, 2nd ed., p. 498. W. B. Saunders, Philadelphia and London.

Neill, C., and Mounsey, P. (1958). Auscultation in patent ductus arteriosus; with a description of two fistulæ simulating patent ductus. Brit. Heart f., 20, 61.

Parker, B. M., Burford, T. H., Carlsson, E. C., and Buchner, E. F. (1963). The diagnosis of aorticopulmonary septal defect. A case report with successful surgical closure. Amer. Heart F., 65, 534.
Reid, J. M., Stevenson, J. G., Coleman, E. N., Barclay, R. S., Welsh, T. M., Fyfe, W. M., and Inall, J. A. (1964). Moderate to severe pulmonary hypertension accompanying patent ductus arteriosus. Brit. Heart F., 26, 600.

Skall-Jensen, J. (1958). Congenital aorticopulmonary fistula. A review of the literature and report of two cases. Acta med. scand., 160, 221.

Straus, F., Lev, M., and Cassels, D. (1964). Clinical pathologic conference. Amer. Heart F., 68, 804.

Taussig, H. B. (1960). Congenital Malformations of the Heart, 2nd ed., pp. 524-535. Vol. II. Specific Malformations. Published for the Commonwealth Fund by Harvard University Press, Cambridge, Mass. and Oxford University Press, London.

Wood, P. (1960). Diseases of the Heart and Circulation, 2nd ed. W. B. Saunders, Philadelphia and London.

The following papers will appear in an early issue of this fournal:

Banding of the Pulmonary Artery for Common Atrio-ventricular Canal. By Jane Somerville, T. Agnew, J. Stark, D. J. Waterston, E. Aberdeen, R. E. Bonham-Carter, and S. Waich.

Right Heart Pressures in Acute Myocardial Infarction. By D. C. Fluck, P. A. Valentine, B. Treister, B. Higgs, D. N. Reid, R. E. Steiner, and J. P. D. Mounsey.

Congenital Complete Heart Block in Pregnancy. By A. C. F. Kenmure and A. J. V. Cameron.

Hæmodynamic Changes During Continuous Exercise. By Edgar Sowton and Felix Burkhart.

Radio-iodine in the Treatment of Congenital Heart Disease with Severe Pulmonary Hypertension. By E. Varnauskas, M. Korsgren, and R. Olander.

Aortic Valve Replacement in the Wolff-Parkinson-White Syndrome. By D. R. Richmond and D. R. Smith.

Hæmodynamic Studies in Shock Associated with Infection. By Henry S. Loeb, Antonio Cruz, Cheng Yee Teng, Jeb Boswell, Raymond J. Pietras, John R. Robin, and Rolf M. Gunnar.

Effect of Propranolol on Left Ventricular Work in Aortic Stenosis. By John Hamer and James Fleming.

Abnormal Electrocardiogram After Adder Bite. By J. S. Chadha, D. W. Ashby, and J. O. Brown.

Papillary "Tumours" of the Left Ventricle. By Donald Heath and Ian McKim Thompson.

Assessment of Reflected Ultrasound as a Diagnostic Instrument in the Study of Mitral Valve Disease. By William L. Winters, Jr., Alcides Riccetto, Jose Gimenez, Michael McDonouth, and Renate Soulen.

A Simple Phonocardiographic Formula for Predicting the Left Atrial Pressure in Mitral Stenosis. By J. P. H. Davies.

Supra-valvar Stenosis of the Pulmonary Artery. By F. G. Schlesinger and G. T. Meester.

Arterial Blood Supply of the Human Sinus Node. By L. Verhaeghe and L. Van der Hauwaert.

Complications in 220 Patients with Cardiac Dysrhythmias Treated by Phased Direct Current Shock, and Indications for Electroconversion. By Leon Resnekov and Lawson McDonald.

Cardio-circulatory Effects of Beta-adrenergic Blockade in Organic Heart Disease. By T. Grandjean and J. L. Rivier.

Vectorcardiographic Evolution of Left Ventricular Hypertrophy. By Hubert Bell, David Pugh, and Marvin Dunn. 